\title{
Factors associated with the burden of family caregivers of elderly patients with femoral neck fracture: a cross-sectional study
}

\author{
Peifen Xiao and Yongchun Zhou*
}

\begin{abstract}
Background: This study aimed to study the factors associated with caregiver burden among caregivers of elderly patients with femoral neck fracture.

Methods: This cross-sectional study was based on a non-probabilistic sampling of 183 elderly postoperative patients (aged 65 years or older) with femoral neck fracture who were hospitalized in the orthopedic center in our hospital and their family caregivers. Data were collected from January 2016 to June 2019. Patients and family caregivers completed the sociodemographic questionnaire. The Social Support Rating Scale (SSRS), the General SelfEfficacy Scale (GSE), and the Chinese version of the Zarit Burden Interview (ZBI) were used to evaluate social support, self-efficacy, and caregiver burden, respectively. By analyzing the clinical data of patients and family caregivers and combining the factors that affect the caregiver burden in parallel studies, we selected the factors that affected the caregiver burden in this study and conducted a multivariate analysis of these factors. $P<0.05$ was considered statistically significant.

Results: We observed 176 caregivers aged $69.28 \pm 7.19$ years old, among whom 52.3\% were male, 58.0\% lived in the city, $84.0 \%$ were spouses of the patients, and $67.0 \%$ had a primary school educational background. The ZBI score of the family caregivers was $37.8 \pm 8.9$, and $82.7 \%$ of the caregivers were under a moderate to severe burden. The patient's functional status, Harris score, and pain score and the caregiver's SSRS scores, GSE scores, and the ratio of medical expenses to monthly income per capita were factors that affected the caregiver burden.
\end{abstract}

Conclusions: Most family caregivers of elderly patients with femoral neck fracture are subject to a considerable care burden, and social support and self-efficacy intervention are conducive to reducing the caregiver burden.

Keywords: Femoral neck fracture, Caregivers, Elderly, Burden, Factors, Hemiarthroplasty

\section{Background}

Femoral neck fracture is an injury of the femoral neck that comes under an external force due to either osteoporosis or hip joint muscle decline [1]. Osteoporosis patients are increasingly common in the elderly population

\footnotetext{
*Correspondence: yong_chunzhou@163.com

Department of Orthopedics, Shaanxi Provincial People's Hospital, Xi'an, Shaanxi, People's Republic of China
}

and present a high-risk group of femoral neck fractures. With an increasing trend of an ageing population in our country, the incidence of femoral neck fracture has increased each year and has become a major disease contributing to a high disability rate, high fatality rate, and high medical costs, thereby seriously affecting the physical and mental health of the elderly $[2,3]$. Hemiarthroplasty (HA) is a common method for treating femoral

(C) The Author(s). 2020 Open Access This article is licensed under a Creative Commons Attribution 4.0 International License, which permits use, sharing, adaptation, distribution and reproduction in any medium or format, as long as you give appropriate credit to the original author(s) and the source, provide a link to the Creative Commons licence, and indicate if changes were made. The images or other third party material in this article are included in the article's Creative Commons licence, unless indicated otherwise in a credit line to the material. If material is not included in the article's Creative Commons licence and your intended use is not permitted by statutory regulation or exceeds the permitted use, you will need to obtain permission directly from the copyright holder. To view a copy of this licence, visit http://creativecommons.org/licenses/by/4.0/ The Creative Commons Public Domain Dedication waiver (http://creativecommons.org/publicdomain/zero/1.0/) applies to the data made available in this article, unless otherwise stated in a credit line to the data. 
neck fracture. HA can significantly improve hip joint function, improve quality of life, and reduce complications $[4,5]$. Rehabilitation management of patients with femoral neck fracture remains a complex and perennial health issue, and nursing for patients with femoral neck fracture calls for long-term caregiver support.

In most areas in China, due to the lack of human resources for nursing and unsound social medical insurance systems, many patients with femoral neck fractures need to be cared for at home. Therefore, the burden of helping patients recover mainly falls on the shoulder of family caregivers, who become the family caregivers of such patients. The concept of a family caregiver refers to a person who has a strong emotional bond with the patient, is a family member, and can provide emotional support and nursing support to the patient during the illness of a family member [6]. Family caregivers play a key role in the management of patients with femoral neck fracture, as they take on the physical, emotional, medical, and financial responsibilities of caring for their sick relatives and thereby experience tremendous pressure and caregiver burden $[7,8]$.

Many factors, including disease-related factors, sociopsychological factors, and clinical and sociodemographic factors, are related to the burden of caregivers [9]. Diseaserelated variables include the patient's functional status, cognitive function, pain score, and Harris score [10-13]. Clinical and sociodemographic factors include variables related to patients and caregivers. Patient-related variables include age, gender, and education level. Caregiver-related variables include age, gender, education level, place of residence, relationship with patient, monthly income, and length of care $[10,11,13]$. Socio-psychological factors encompass social support and self-efficacy [10, 13, 14]. In China, little literature is available on factors related to the burden of caregivers for patients with femoral neck fracture. Concerning the burden of caregivers, understanding specific factors affecting the burden of caregivers, and satisfying the needs of caregivers can, on the one hand, lessen the pressure on caregivers, enhance their care and nursing skills, and speed up patient recovery; on the other hand, they can support medical and health institutions and the government to improve the current situation of care for patients with femoral neck fracture. Therefore, this study analyzed and evaluated the clinical data of patients and their family caregivers, combined with related factors affecting the caregiver burden in the relevant literature, for the purpose of clarifying the factors that affect the burden of family caregivers for elderly patients with femoral neck fracture.

\section{Methods}

\section{Study participants}

We investigated 183 family caregivers of elderly patients aged $\geqslant 65$ years undergoing HA for a femoral neck fracture. A cross-sectional study was conducted. We employed non-probability sampling methods to select participants. We included any family members aged between 18 and 75 who had lived with and cared for an elderly patient with a femoral neck fracture for longer than 3 months, who were able to speak, read, and understand Chinese, and who were willing to participate in the study. We excluded those who suffered from a mental disorder; those with communication difficulties or difficulty understanding the survey questions; those who had cognitive dysfunction; those who did not sign a written informed consent form; those who had cared for patients for less than 3 months; and those who declined to participate in the study or withdrew halfway. The ethics review committee of the Shaanxi Provincial People's Hospital approved the study protocol. All the patients and caregivers provided written informed consent for the use and publication of data for research purposes.

\section{Data collection}

The data were collected from Shaanxi Provincial People's Hospital by trained personnel, under the direction of the first author, between January 2016 and June 2019. We conducted a sociodemographic questionnaire of patients with femoral neck fracture and their family caregivers. The demographic data collected from patients included age, gender and education level. The demographic data collected from family caregivers included age, gender, education level, place of residence, relationship with patient, duration of care, and the ratio of medical expenses to monthly income per capita. We obtained the patients' clinical data from their medical records, such as the fracture site, functional status, pain level, and Harris score. After the researcher explained the study's objectives, methods, benefits, and potential risks to family caregivers, those who agreed to participate in the study signed a written informed consent form. Subsequently, we conducted a face-to-face interview with them.

\section{Evaluation standard}

The burden of family caregivers was assessed using the Zarit Burden Interview (ZBI) [15]. The Chinese version of the ZBI was reliable and valid for Chinese caregivers [16]. The total score of care burden can be divided into 3 levels: $0-20$ indicates little or no burden; $21-39$ indicates a medium burden; and 40-88 indicates a severe burden. The scale's overall internal consistency is very high (Cronbach's alpha $=0.88)$ [13].

Social support is measured using the Social Support Rating Scale (SSRS) [17], which consists of 3 subscales with 10 items. A higher score indicates a higher level of social support. The scale's overall internal consistency is very high (Cronbach's alpha $=0.90)[13]$. 
The self-efficacy of caregivers was measured using the General Self-Efficacy Scale (GSE) [18]. A higher score indicates a higher self-efficacy. The scale's overall internal consistency is very high (Cronbach's alpha $=0.91$ ) [13].

\section{Statistical analysis}

The data were processed using SPSS 19.0 statistical software (SPSS, IL, USA). Enumeration data were expressed as percentages (\%), and measurement data were expressed as " $x \pm s$ ". Spearman's rank correlation coefficient was used to calculate the correlation. Single variables and important factors in the correlation analysis were entered into a stepwise multiple linear regression, in which the caregiver burden was used as a dependent variable. $P<0.05$ was considered statistically significant.

\section{Results}

The study recruited 183 caregivers in total, 2 of whom refused to participate and 5 of whom quit halfway. Ultimately, the sample size (with a response rate of $96.2 \%$ ) was 176.

\section{Characteristics of the patients and caregivers}

A total of 176 patients with femoral neck fracture ranged from 21 to 79 years of age (with a mean $68.15 \pm 6.37$ years), and $54.0 \%$ were female. A total of $63.1 \%$ of the patients had an educational background of primary school or below, $12.5 \%$ of the patients were dependent on daily activities, $44.3 \%$ had moderate or severe pain, $80.7 \%$ had a Harris score greater than 70 , and $27.2 \%$ had a bilateral femoral neck fracture (Table 1).

A total of 176 caregivers were all from the patients' families, ranging from 21 to 79 years of age (with a mean of $69.28 \pm 7.19$ years). A total of $67.0 \%$ of them had an education of primary school or below, and most of them lived in urban areas (58\%). A total of $84.1 \%$ of the caregivers were patients' spouses. A total of $35.2 \%$ of caregivers took care of patients for more than 3 months, and $75.6 \%$ of them had a ratio of medical expenses to monthly income per capita of less than 5/1 (Table 1).

\section{Correlations of caregiver burden with the SSRS and GSE scores}

The average ZBI score of family caregivers was $37.8 \pm$ 8.9. Among them, $17.3 \%$ of the caregivers had a ZBI rating of little or no burden, $62.9 \%$ had a rating of moderate burden, and $19.8 \%$ had a severe burden. The average SSRS score of family caregivers was $41.9 \pm 7.2$, and the average GSE score was $26.1 \pm 6.9$. This study analyzed the correlations between ZBI, SSRS, and GSE scores to assess the relationship between caregiver burden and self-efficacy and social skills. The analysis results showed a negative correlation between ZBI scores and SSRS and GSE scores.
Table 1 Demographic and clinical characteristics of patients and their primary caregivers

\begin{tabular}{|c|c|c|}
\hline Variables & Patients $(n=176)$ & Caregivers $(n=176)$ \\
\hline Age (years old) & $68.15 \pm 6.37$ & $69.28 \pm 7.19$ \\
\hline \multicolumn{3}{|l|}{ Gender } \\
\hline Female & 95 (54.0\%) & $84(47.7 \%)$ \\
\hline Male & $81(46.0 \%)$ & $92(52.3 \%)$ \\
\hline \multicolumn{3}{|l|}{ Educational level } \\
\hline$\leqq$ Primary school & $111(63.1 \%)$ & $118(67.0 \%)$ \\
\hline$\geqq$ Secondary school & 65 (36.9\%) & $58(33.0 \%)$ \\
\hline \multicolumn{3}{|l|}{ Place of residence } \\
\hline Countryside & N/A & 74 (42.0\%) \\
\hline City & N/A & $102(58.0 \%)$ \\
\hline \multicolumn{3}{|l|}{ Relationship } \\
\hline Spouse & N/A & $148(84.1 \%)$ \\
\hline Other & N/A & $28(15.9 \%)$ \\
\hline \multicolumn{3}{|l|}{ Care time } \\
\hline$<3$ months & N/A & $114(64.8 \%)$ \\
\hline$>3$ months & N/A & $62(35.2 \%)$ \\
\hline \multicolumn{3}{|c|}{ The ratio of medical expenses and monthly income per capita } \\
\hline$<10 / 1$ & N/A & $28(15.9 \%)$ \\
\hline $10 / 1-5 / 1$ & N/A & $105(59.7 \%)$ \\
\hline$>5 / 1$ & N/A & $43(24.4 \%)$ \\
\hline \multicolumn{3}{|l|}{ Fracture site } \\
\hline Left side & $61(34.7 \%)$ & N/A \\
\hline Right side & $67(38.1 \%)$ & N/A \\
\hline Both sides & $48(27.2 \%)$ & N/A \\
\hline \multicolumn{3}{|c|}{ Functional status (activities of daily living) [12] } \\
\hline Fully independent & $65(36.9 \%)$ & N/A \\
\hline Fully dependent & $22(12.5 \%)$ & N/A \\
\hline Other & $89(50.6 \%)$ & N/A \\
\hline \multicolumn{3}{|l|}{ Harris score [13] } \\
\hline$\geqq 90$ & $102(58.0 \%)$ & N/A \\
\hline $70-89$ & $40(22.7 \%)$ & N/A \\
\hline$<70$ & 34 (19.3\%) & N/A \\
\hline \multicolumn{3}{|l|}{ Pain level, VAS score } \\
\hline$\leqq 3$ & $98(55.7 \%)$ & N/A \\
\hline $4-6$ & $51(29.0 \%)$ & N/A \\
\hline$\geqq 7$ & $27(15.3 \%)$ & N/A \\
\hline
\end{tabular}

Values are presented as the mean and standard deviation or as the number and percentage.

VAS visual analog scale

\section{Factors associated with caregiver burden Univariate analysis}

The univariate analysis showed that the care burden was not related to the patient's age, gender, education level, fracture site, or other characteristics but rather was 
significantly related to the patient's functional status, Harris score, and pain level (Table 2).

The univariate analysis indicated that caregiver burden was not associated with the caregiver's age, gender, education level, relationship with the patient, and other characteristics but rather was significantly correlated with the caregiver's place of residence, care duration, and the ratio of medical expenses to monthly income per capita (Table 3 ).

\section{Multivariate analysis}

The multivariate analysis revealed that caregiver burden was positively correlated with the patient's functional status, Harris score, pain score, GSE score, SSRS score, and the ratio of medical expenses to monthly income per capita. These identified factors could explain approximately $60 \%$ of the variation in caregiver burden.

\section{Discussion}

HA is conducive to improving joint function recovery and reducing complications for patients with femoral neck fracture, and it has been widely applied clinically as a surgical method to treat elderly patients with femoral neck fracture. This study analyzed the clinical data of elderly patients with femoral neck fracture and that of their family caregivers. Combined with reported factors that affect the caregiver burden, this study analyzed the burden-related factors of caregivers of patients who underwent an operation after femoral neck fracture and primarily revealed the burden borne by caregivers who cared for patients with femoral neck fracture in this area of China. This study showed that most of the family caregivers of patients with femoral neck fracture came under considerable caregiver burden, and the important factors that were obviously correlated with the care burden were the patient's functional status, Harris score, pain score, GSE score, SSRS score, and the ratio of medical expenses to monthly income per capita.

In this study, the sociodemographic characteristics of caregivers indicated that family caregivers were the patients' spouses, who had a low education level and were advanced in age. However, these demographic characteristics did not aggravate the caregiver burden of caregivers. This is consistent with the results of related parallel studies [10], which may be due to the similar sociodemographic characteristics of caregivers in Western China as well as the consistency in cultural or behavioral factors. This study showed that the patient's functional status was positively correlated with the caregiver burden. Patients with femoral neck fracture need increased care and personal nursing in their daily lives due to the partial or total loss of self-care ability after the operation, thereby increasing the care burden. This study also showed that the degree of pain that patients experienced was positively correlated with the burden of care. Severe pain affected the quality of life of patients

Table 2 Univariate analysis of caregiver burden and patient variables

\begin{tabular}{|c|c|c|c|c|}
\hline Variable & Little/no burden & Medium burden & Serious burden & Statistical significance \\
\hline Age (years old) & $67.23 \pm 8.13$ & $70.27 \pm 7.83$ & $69.57 \pm 7.86$ & $P>0.05$ \\
\hline \multicolumn{5}{|l|}{ Gender } \\
\hline Female & $17(56.7 \%)$ & $61(55.0 \%)$ & $17(48.6 \%)$ & $P>0.05$ \\
\hline Male & $13(43.3 \%)$ & $50(45.0 \%)$ & $18(51.4 \%)$ & $P>0.05$ \\
\hline \multicolumn{5}{|l|}{ Educational level } \\
\hline$\leqq$ Primary school & $20(66.7 \%)$ & $69(62.2 \%)$ & $22(62.9 \%)$ & $P>0.05$ \\
\hline$\geqq$ Secondary school & $10(33.3 \%)$ & $42(37.8 \%)$ & $13(37.1 \%)$ & $P>0.05$ \\
\hline \multicolumn{5}{|l|}{ Fracture site } \\
\hline Bilateral & $8(26.7 \%)$ & $30(27.0 \%)$ & $10(28.6 \%)$ & $P>0.05$ \\
\hline Other & $22(73.3 \%)$ & $81(73.0 \%)$ & $25(71.4 \%)$ & $P>0.05$ \\
\hline \multicolumn{5}{|c|}{ Functional status (activities of daily living) } \\
\hline Fully independent & $11(36.7 \%)$ & $41(36.9 \%)$ & $13(37.1 \%)$ & $P<0.05$ \\
\hline Other & $19(63.3 \%)$ & $70(63.1 \%)$ & $22(62.9 \%)$ & $P<0.05$ \\
\hline \multicolumn{5}{|l|}{ Harris score [14] } \\
\hline$\geqq 70$ & $24(80.0 \%)$ & $90(81.1 \%)$ & $28(80.0 \%)$ & $P<0.05$ \\
\hline$<70$ & $6(20.0 \%)$ & $21(18.9 \%)$ & 7 (20.0\%) & $P<0.05$ \\
\hline \multicolumn{5}{|l|}{ Pain level, VAS score } \\
\hline$\leqq 3$ & $17(56.7 \%)$ & $62(55.9 \%)$ & 19 (54.3\%) & $P<0.05$ \\
\hline$>4$ & 13 (43.3\%) & 49 (44.1\%) & 16 (45.7\%) & $P<0.05$ \\
\hline
\end{tabular}


Table 3 Univariate analysis of caregiver burden and caregiver variables

\begin{tabular}{|c|c|c|c|c|}
\hline Variable & Little/no burden & Medium burden & Severe burden & Statistical significance \\
\hline Age (years old) & $70.12 \pm 6.45$ & $71.45 \pm 4.01$ & $68.10 \pm 7.65$ & $P>0.05$ \\
\hline \multicolumn{5}{|l|}{ Gender } \\
\hline Female & $14(46.7 \%)$ & $53(47.7 \%)$ & $17(48.6 \%)$ & $P>0.05$ \\
\hline Male & $16(53.3 \%)$ & $58(52.3 \%)$ & $18(51.4 \%)$ & $P>0.05$ \\
\hline \multicolumn{5}{|l|}{ Educational level } \\
\hline$\leqq$ Primary school & $21(70.0 \%)$ & $74(66.6 \%)$ & $23(65.7 \%)$ & $P>0.05$ \\
\hline$\geqq$ Secondary school & $9(30.0 \%)$ & 37 (33.3\%) & $12(34.3 \%)$ & $P>0.05$ \\
\hline \multicolumn{5}{|l|}{ Place of residence } \\
\hline Countryside & $12(40.0 \%)$ & $47(60.3 \%)$ & $15(61.5 \%)$ & $P<0.05$ \\
\hline City & $18(60.0 \%)$ & $64(39.7 \%)$ & $20(38.5 \%)$ & $P<0.05$ \\
\hline \multicolumn{5}{|l|}{ Relationship } \\
\hline Spouse & $25(83.3 \%)$ & $94(84.7 \%)$ & $29(82.9 \%)$ & $P>0.05$ \\
\hline Other & $5(16.7 \%)$ & $17(15.3 \%)$ & $6(17.1 \%)$ & $P>0.05$ \\
\hline \multicolumn{5}{|l|}{ Care duration } \\
\hline$<9$ months & 19 (63.3\%) & 72 (64.9\%) & $23(65.7 \%)$ & $P<0.05$ \\
\hline$>9$ months & $11(36.7 \%)$ & 39 (35.1\%) & $12(34.3 \%)$ & $P<0.05$ \\
\hline \multicolumn{5}{|c|}{ The ratio of medical expenses to monthly income per capita } \\
\hline$\leqq 5 / 1$ & $23(76.7 \%)$ & $84(75.7 \%)$ & $26(74.3 \%)$ & $P<0.05$ \\
\hline$>5 / 1$ & 7 (23.3\%) & 27 (24.3\%) & 9 (25.7\%) & $P<0.05$ \\
\hline
\end{tabular}

and was injurious to their body and mind. In addition, pain requires continuous analgesic therapy as well as sustained emotional support, which not only increases the caregiver's expenditure but also contributes to mental health problems in the caregiver and ultimately adds to the burden of care. In this study, most of the family caregivers had a ratio of medical expenses to monthly income per capita of less than 5/1. As a result, these caregivers experienced enormous life pressures, heightening the burden of caregivers. In summary, caregivers had to pay medical expenses and support the family while caring for patients. They were under tremendous pressure physically, psychologically, and economically. The persistent stress load affected the health of caregivers, and the health status of caregivers also directly influenced patients' rehabilitation and quality of life. Therefore, it is necessary for health care institutions and government to introduce specific measures and policies to reduce the care burden for the families of elderly caregivers, such as hospital strengthening care skills training. Additionally, through refining the rehabilitation training guidance and increasing emotional support, the government increases the provision and support for the aged, thus increasing financial support and reducing medical expenses.

Previous studies have demonstrated that caregivers' social support and self-efficacy are negatively correlated with caregiver burden $[17,18]$. Caregivers feel that social support affects their anxiety and depression levels, as it can effectively lessen the severity of their stress [19]. However, when the targeted support provided by professionals to caregivers is not satisfactory, the pressure felt by caregivers augments [20]. This study shows that social support can lessen caregiver burden, consistent with the above studies. In China, due to the influence of traditional culture and a lack of available services (for example, in single-child families, offspring cannot take care of elderly parents due to work reasons), caregivers often do not receive substantial support from other members of the family, while social support from the family and professional institutions makes it easier for family caregivers to take care of patients and themselves, cope with stress, and minimize the caregiver burden [21, 22]. Therefore, to reduce the burden of caregivers, professional medical and health institutions can take measures to improve the current situation, provide appropriate social support to help caregivers, and reduce their burden. Self-efficacy is the individual's confidence or belief in a new organization and its executive capability in a particular field. Individuals with high self-efficacy will carry out the task more proactively, while individuals with low self-efficacy may evade it due to a sense of inability and helplessness [23]. Self-efficacy can bring about more positive emotions and contribute to health [24], thereby reducing the caregiver burden [25]. The results of the questionnaire in this study reveal that self-efficacy is 
negatively correlated with caregiver burden, consistent with the above results, possibly because family caregivers with high self-efficacy have strong self-regulation abilities, are psychologically healthier, and can better cope with care pressure [26]. Specifically, individuals with a strong ability in emotion regulation can adjust their emotions to fit in with the situation they are in at the moment. Generally, they have more positive emotional experiences, which can in turn promote their mental health and enhance their happiness, make them more flexible in thought and encourage them to come up with solutions to the problem. Given our research findings, more intervention measures should be taken to improve the self-efficacy of family caregivers. Intervention measures can include professional trainings or psychological support, which may help reduce the burden of family caregivers of elderly patients with femoral neck fracture.

This study also has some limitations, as it only involves an orthopedic center in Western China. Considering the cross-sectional nature of this study, it is not plausible to establish causality between the results and the survey variables. To better understand the factors influencing the caregiver burden among family caregivers of patients with femoral neck fracture in China, it is also necessary to conduct a longitudinal cohort study to gain deeper insight into the factors involved in the care burden of family caregivers and the impact of these factors on caregivers. In addition, the inclusion of nonrandomly selected participants may lead to unpredictable biases. Despite the above limitations, this study has identified factors closely related to the caregiver burden and has proposed improvement strategies and intervening measures, which will eventually contribute to better patient care.

\section{Conclusion}

This study focuses on the caregiver burden among family caregivers of patients with femoral neck fracture. Family caregivers of patients with femoral neck fracture usually have a poor quality of life and experience tremendous psychological and economic pressure. Most of them are subject to a heavy burden. The burden of the caregiver is directly related to the patient's functional status, pain score, Harris score, GSE score, SSRS score, and the ratio of medical expenses to monthly income per capita, while it is negatively correlated with social support and self-efficacy. Patients with femoral neck fracture should be given more attention. Meanwhile, social support should be provided to family caregivers, and efforts should be made to improve their self-efficacy.

\section{Abbreviations}

SSRS: Social Support Rating Scale; ZBI: Zarit Burden Interview; GSE: General Self-Efficacy Scale; SD: Standard deviation

\section{Acknowledgements}

Not applicable.

\section{Authors' contributions}

YZ and PX participated in the recruitment, data collection, and analysis. All the authors contributed to the study design and drafting of the manuscript. All the authors read and approved the final manuscript.

\section{Funding}

Not applicable.

\section{Availability of data and materials}

The datasets supporting the conclusions of this article are included within the article. The raw data can be requested from the corresponding author on reasonable request.

\section{Ethics approval and consent to participate}

Ethical approval from the Ethics Committee of Shaanxi Provincial People's Hospital was obtained for this study. Each author certifies that all the investigations were conducted in conformity with ethical principles. Written informed consent was obtained from all participants, caregivers, and patients included in the study.

\section{Consent for publication}

All patients and caregivers consented to the publication of their personal data in this article.

\section{Competing interests}

The authors declare that they have no competing interests.

Received: 18 March 2020 Accepted: 16 June 2020

Published online: 23 June 2020

\section{References}

1. Stevens JA, Rudd RA. The impact of decreasing U.S. hip fracture rates on future hip fracture estimates. Osteoporos Int. 2013;24(10):2725-8.

2. Boylan MR, Riesgo AM, Paulino CB, Tejwani NC. Day of admission is associated with variation in geriatric hip fracture care. J Am Acad Orthop Surg. 2019;27(1):e33-40.

3. Liodakis E, Antoniou J, Zukor DJ, et al. Major complications and transfusion rates after hemiarthroplasty and total hip arthroplasty for femoral neck fractures. J Arthroplast. 2016;31(9):2008-12.

4. van den Bekerom MP, Hilverdink EF, Sierevelt IN, et al. A comparison of hemiarthroplasty with total hip replacement for displaced intracapsular fracture of the femoral neck: a randomised controlled multicentre trial in patients aged 70 years and over. J Bone Joint Surg Br. 2010;92(10):1422-8.

5. Avery PP, Baker RP, Walton MJ, et al. Total hip replacement and hemiarthroplasty in mobile, independent patients with a displaced intracapsular fracture of the femoral neck: a seven- to ten-year follow-up report of a prospective randomised controlled trial. J Bone Joint Surg Br. 2011;93(8):1045-8.

6. Toledano-Toledano F, Contreras-Valdez JA. Validity and reliability of the Beck depression inventory II (BDI-II) in family caregivers of children with chronic diseases. PLoS One. 2018, 28, 13(11):e0206917.

7. Boylan MR, Riesgo AM, Paulino CB, Tejwani NC. Is patient satisfaction associated with objective measures of geriatric hip fracture care? Bull Hosp Jt Dis (2013). 2018;76(4):252-8.

8. Raaymakers EL. Fractures of the femoral neck: a review and personal statement. Acta Chir Orthop Traumatol Cechoslov. 2006;73(1):45-59.

9. Yu Y, Liu ZW, Tang BW, Zhao M, Liu XG, Xiao SY. Reported family burden of schizophrenia patients in rural China. PLoS One. 2017;16(6):e0179425.

10. Li C, Miao J, Gao X, Zheng L, Su X, Hui H, et al. Factors associated with caregiver burden in family caregivers of patients with adolescent scoliosis: a descriptive cross-sectional study. Med Sci Monit. 2018;24:6472-9.

11. Saria MG, Courchesne N, Evangelista L, Carter J, MacManus DA, Gorman MK, et al. Cognitive dysfunction in patients with brain metastases: influences on caregiver resilience and coping. Support Care Cancer. 2017;25(4):1247-56.

12. Linacre JM, Heinemann AW, Wright BD, Granger CV, Hamilton BB. The structure and stability of the functional independence measure. Arch Phys Med Rehabil. 1994;75:127-32. 
13. Luo J, Zhou Y, Liu H, Hu J. Factors related to the burden of family caregivers of elderly patients with spinal Tumours in Northwest China. BMC Neurol. 2020;20(1):69.

14. Souza ALR, Guimarães RA, de Araújo VD, de Assis RM, de Almeida Cavalcante Oliveira LM, Souza MR, et al. Factors associated with the burden of family caregivers of patients with mental disorders: a cross-sectional study. BMC Psychiatry. 2017;17(1):353.

15. Orr NK, Zarit JM, Zarit SH. The hidden victims of Alzheimer's disease: families under stress. New York: New York University Press; 1985.

16. Lu L, Wang L, Yang $X$, et al. Zarit caregiver burden interview: development, reliability and validity of the Chinese version. Psychiatry Clin Neurosci. 2009; 63:730-4.

17. Xiao SY. Theoretical foundation and research application about the social support rating scale. J Clin Psychiatry. 1994;4:98-100.

18. Romero-Moreno R, Losada A, Mausbach BT, Márquez-González M, Patterson TL, López J. Analysis of the moderating effect of self-efficacy domains in different points of the dementia caregiving process. Aging Ment Health. 2011;15(2):221-31.

19. Hileman JW, Lackey NR. Self-identified needs of patients with cancer at home and their home caregivers:a descriptive study. Oncol Nurs Forum. 1990;17(6):907-13.

20. Sharpe L, Butow P, Smith C, et al. The relationship between available support, unmet needs and caregiver burden in patients with advanced cancer and their carers. Psychooncology. 2005;14(2):102-14.

21. Gong G, Mao J. Health-related quality of life among chinese patients with rheumatoid arthritis: the predictive roles of fatique, functional disability, selfefficacy, and social support. Nurs Res. 2016;65(1):55-67.

22. Hu X, Hu X, Su Y, Qu M. Quality of life among primary family caregivers of patients with heart failure in Southwest China. Rehabil Nurs. 2018;43(1):26-34.

23. Bandura A. Self-efficacy oward a unifying theory of behaviod change. PsycholoRev. 1997;84(3):191-215.

24. Tang WK, Chan CY. Effects of psychosocial interventions on self-efficacy of dementia caregivers: a literature review. Int J Geriatr Psychiatry. 2016;31(5): 475-93.

25. Pinquart M, Sörensen S. Associations of stressors and uplifts of caregiving with caregiver burden and depressive mood: a meta-analysis. J Gerontol B Psychol Sci Soc Sci. 2003;58(2):112-28.

26. Silvers JA, McRae K, Gabrieli JDE. Age-related differences in emotional reactivity, regulation, and rejection sensitivity in adolescence. Emotion. 12(6): 1235-47.

\section{Publisher's Note}

Springer Nature remains neutral with regard to jurisdictional claims in published maps and institutional affiliations.

Ready to submit your research? Choose BMC and benefit from:

- fast, convenient online submission

- thorough peer review by experienced researchers in your field

- rapid publication on acceptance

- support for research data, including large and complex data types

- gold Open Access which fosters wider collaboration and increased citations

- maximum visibility for your research: over $100 \mathrm{M}$ website views per year

At BMC, research is always in progress.

Learn more biomedcentral.com/submissions 